\title{
Transient Hyperkalemia Following Treatment of Chronic Hypokalemia: A Case Report and Review of Distal Tubule Physiology
}

\author{
Matthew C. Breeggemann Stephen L. Gluck \\ Division of Nephrology, University of California San Francisco, San Francisco, CA, USA
}

\section{Keywords}

Hypokalemia - Hyperkalemia - With no lysine kinases · Ste20-related proline-alanine-rich kinase $\cdot$ Oxidative stress-responsive kinase-1

\begin{abstract}
Hypokalemia is a relatively common electrolyte disorder usually resulting from gastrointestinal wasting. Transient hyperkalemia in those treated for hypokalemia has been previously described to occur in $16 \%$ of hospitalized patients. The majority of those patients had acute, hospital-acquired hypokalemia. Here, we report a case of a young man with alcohol use disorder and chronic hypokalemia who was hospitalized for muscle weakness, abdominal pain, and intractable emesis. His potassium was $2.5 \mathrm{mEq} / \mathrm{L}$ on the day of admission. Four days later, with a creatinine at baseline $(0.9 \mathrm{mg} / \mathrm{dL})$, potassium abruptly increased to $6.7 \mathrm{mEq} / \mathrm{L}$. He did not have evidence of hyperaldosteronism. In cases of chronic hypokalemia, we propose that the adaptive mechanisms of the distal tubule with total body potassium deficits require time to revert back to a nonactive state and that transient hyperkalemia may be observed during these "refractory" periods during which potassium supplementation is continued. The time required for disassembly of with no lysine kinases following resolution of hypokalemia is unknown. Hyperkalemia is an important consideration when treating patients with chronic hypokalemia.
\end{abstract}

\section{Karger $\stackrel{N}{\%}$}




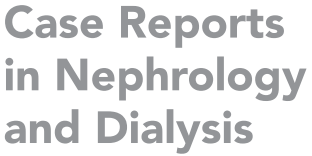

Introduction

\begin{tabular}{|c|c|}
\hline \multicolumn{2}{|c|}{ Case Rep Nephrol Dial 2022;12:1-4 } \\
\hline DOI: 10.1159/000521477 & $\begin{array}{l}\text { (c) } 2022 \text { The Author(s). Published by S. Karger AG, Basel } \\
\text { www.karger.com/cnd }\end{array}$ \\
\hline
\end{tabular}

Electrolyte disorders are common among hospitalized patients. In a recent study of over 322,000 patients, hyponatremia (16.6\%) and hypokalemia $(13.7 \%)$ were reported as the most prevalent [1]. Furthermore, $16 \%$ of hospitalized patients with hypokalemia were found to experience transient hyperkalemia during treatment. Most of those patients suffered from hospital-acquired hypokalemia and had associated hypomagnesemia [2]. The etiology of transient hyperkalemia is unknown, and there may be differences between those with acute versus chronic hypokalemia. In chronic hypokalemia, low serum potassium is rarely associated with decreased dietary intake alone due to renal compensatory mechanisms including those involving the distal tubule epithelial cells. As previously reported, with no lysine (WNK) kinases complex with Ste20-related proline-alanine-rich kinase and oxidative stress-responsive kinase-1 to form WNK bodies [3]. These complexes are more likely to aggregate in hypokalemic states due to cellular hyperpolarization and subsequent decrease in the intracellular chloride concentration from exit of chloride anions via the basolateral Cl-channel $\mathrm{Kb}$ [4]. Intracellular chloride normally suppresses WNK4 activity and thus impairs aggregation with WNK1, Ste20related proline-alanine-rich kinase, and oxidative stress-responsive kinase-1 [4,5]. Furthermore, kidney-specific WNK1 knockout animal models on low potassium diets have impaired WNK body formation [6]. WNK bodies are thought to increase the activity of the sodium-chloride cotransporter leading to decreased sodium delivery to the epithelial sodium channel found in the principal cells. This process is critical in hypokalemic states as it results in decreased urinary potassium wasting through the renal outer medullary potassium channel. The amount of time required for disassembly of WNK bodies following resolution of hypokalemia is unknown.

\section{Case Report}

A 30-year-old man with chronic alcoholic pancreatitis, alcohol use disorder, and opiate use disorder was hospitalized for muscle weakness, abdominal pain, and intractable emesis. He reported alcohol use usually amounting to one pint of vodka daily. His last drink was the day of admission. He was not prescribed any medications but did occasionally take nonprescribed oxycodone. His abdominal pain was epigastric. Lipase was 59 U/L (7-58). The muscle weakness was poorly described although acutely started $2 \mathrm{~h}$ prior to presentation and involved both of his legs. Serum potassium was $2.5 \mathrm{mEq} / \mathrm{L}$ (3.5-5.1). Venous blood gas was notable for a pH of 7.54 (7.32-7.42). Serum bicarbonate was $41 \mathrm{mmol} / \mathrm{L}$ (22-29). He denied any family history of electrolyte disorders. Thyroid-stimulating hormone, checked to evaluate for hypokalemic periodic paralysis, was $2.46 \mathrm{mU} / \mathrm{mL}(0.37-4.42)$. Magnesium levels were normal. Serum creatinine was $1.5 \mathrm{mg} / \mathrm{dL}$ although quickly improved to baseline of approximately 0.9 over the next several days. He was treated for alcohol withdrawal using oral benzodiazepines and for hypokalemia using a combination of intravenous and oral potassium. All of his symptoms gradually improved. On the fourth day of the hospitalization, his serum potassium was $6.7 \mathrm{mEq} / \mathrm{L}$ (no hemolysis, recheck $6.4 \mathrm{mEq} / \mathrm{L}$ ). Serum bicarbonate had normalized to $26 \mathrm{mmol} / \mathrm{L}$. Excluding diet, which was minimal due to his gastrointestinal symptoms, he had received approximately $340 \mathrm{mEq}$ of potassium prior to this critical lab result. Shown in Figure 1 is a summary of his potassium levels and total daily supplemental potassium received. He was treated with loop diuretics and potassium binders. $24 \mathrm{~h}$ urine potassium was $35 \mathrm{mEq} / \mathrm{L}$. He had a normal aldosterone concentration $(5.8 \mathrm{ng} / \mathrm{dL},<17)$ and renin activity $(0.3 \mathrm{ng} / \mathrm{mL} / \mathrm{h}, 0.2-1.6)$. Prior abdominal computed tomography imaging demonstrated normal-appearing adrenal glands and kidneys. His serum potassium remained normal for the remainder of the hospitalization, and he was discharged after 10 days.

\section{Karger's}


Case Reports in Nephrology and Dialysis

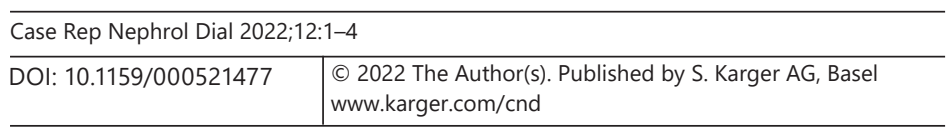

Breeggemann and Gluck: Transient Hyperkalemia following Treatment of Chronic Hypokalemia
Fig. 1. Serum potassium results graphed by hospital day. Estimated daily $\mathrm{mEq}$ of supplemental potassium received is listed above corresponding lab values.

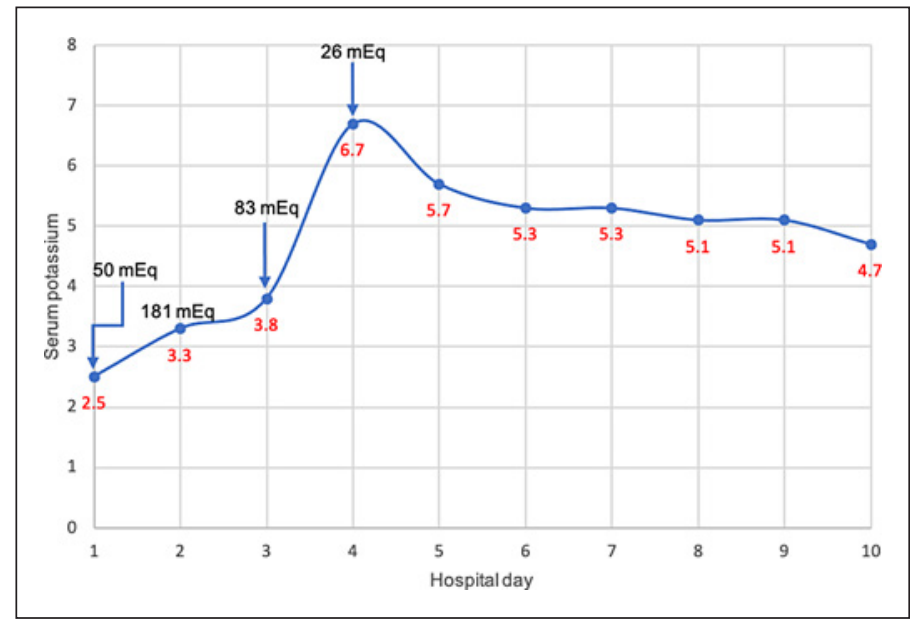

\section{Discussion}

This patient suffered from chronic potassium depletion likely due to poor dietary intake and gastrointestinal wasting both from alcohol use disorder. Serum potassium was usually low as part of outpatient data available in electronic medical records. The transient hyperkalemia he experienced during his hospitalization has been previously reported in a young woman with chronic hypokalemia from intentional ingestion of Hinkle's Cascara Compound and in a minority of patients described in an observational study [2, 7]. It is unknown why some patients develop transient hyperkalemia but risk factors are thought to include higher (but not more frequent) administered doses of potassium, concurrent magnesium supplementation, concurrent total parenteral nutrition, and those with hematological malignancies [2]. We propose a possible mechanism for the transient hyperkalemia seen in chronic hypokalemia cases. The disassembly and inactivation of WNK bodies may not be instantaneous when normal serum potassium levels are achieved in those with chronic potassium deficits. Continued treatment with potassium-containing agents may cause transient hyperkalemia in some patients due to a temporary impairment in the ability of the principal cells to excrete potassium. Identifying those at high risk for this potentially life-threatening electrolyte abnormality, obtaining basic metabolic panels several times daily, and promptly treating with cessation of additional potassium supplements and use of potassium lowering medications are all important considerations in those receiving treatment for hypokalemia.

\section{Acknowledgments}

Publication made possible in part by support from the UCSF Open Access Publishing Fund.

\section{Statement of Ethics}

This research work does not contain human or animal subject research material. This review of patient data did not require ethical approval in accordance with local/national guidelines. Written informed consent was obtained from the patient for publication of this case report and any accompanying images. 
Case Reports

in Nephrology

and Dialysis

\begin{tabular}{l|l}
\hline Case Rep Nephrol Dial 2022;12:1-4 \\
\hline DOI: 10.1159/000521477 & $\begin{array}{l}\text { @ 2022 The Author(s). Published by S. Karger AG, Basel } \\
\text { www.karger.com/cnd }\end{array}$ \\
\hline
\end{tabular}

Breeggemann and Gluck: Transient Hyperkalemia following Treatment of Chronic Hypokalemia

\section{Conflict of Interest Statement}

The authors have no conflicts of interest to declare.

\section{Funding Sources}

This research work was performed without any funding.

\section{Author Contributions}

Matthew C. Breeggemann was involved with direct patient care, chart reviewing the case, performing literature review, and writing the manuscript. Stephen L. Gluck was involved with performing literature review and editing the manuscript.

\section{Availability of Data and Materials}

Research data are not publicly available as it is contained within a confidential medical record.

\section{References}

1 Wang Y, Hu J, Geng X, Zhang X, Xu X, Lin J, et al. A novel scoring system for assessing the severity of electrolyte and acid-base disorders and predicting outcomes in hospitalized patients. J Investig Med. 2019;67(4):750-60.

2 Crop MJ, Hoorn EJ, Lindemans J, Zietse R. Hypokalaemia and subsequent hyperkalaemia in hospitalized patients. Nephrol Dial Transplant. 2007 Dec;22(12):3471-7.

3 Terker AS, Zhang C, McCormick JA, Lazelle RA, Zhang C, Meermeier NP, et al. Potassium modulates electrolyte balance and blood pressure through effects on distal cell voltage and chloride. Cell Metab. 2015 Jan;21(1):39-50.

4 Thomson MN, Cuevas CA, Bewarder TM, Dittmayer C, Miller LN, Si J, et al. WNK bodies cluster WNK4 and SPAK/OSR1 to promote NCC activation in hypokalemia. Am J Physiol Renal Physiol. 2020 Jan;318(1):F216-28.

5 Hoorn EJ, Gritter M, Cuevas CA, Fenton RA. Regulation of the renal NaCl cotransporter and its role in potassium homeostasis. Physiol Rev. 2020 Jan;100(1):321-56.

6 Boyd-Shiwarski CR, Shiwarski DJ, Roy A, Namboodiri HN, Nkashama LJ, Xie J, et al. Potassium-regulated distal tubule WNK bodies are kidney-specific WNK1 dependent. Mol Biol Cell. 2018 Feb;29(4):499-509.

7 Schwartz WB, Relman AS. Metabolic and renal studies in chronic potassium depletion resulting from overuse of laxatives. J Clin Invest. 1953 Mar;32(3):258-71.

\section{Karger'}

